PROCEEDINGS OF THE

AMERICAN MATHEMATICAL SOCIETY

Volume 128, Number 9, Pages 2745-2748

S 0002-9939(00)05762-2

Article electronically published on April 27, 2000

\title{
A NOTE ON DUALITY BETWEEN MEASURE AND CATEGORY
}

\author{
TOMEK BARTOSZYŃSKI \\ (Communicated by Alan Dow)
}

Abstract. We show that there is no Erdös-Sierpiński mapping preserving addition.

Let $\mathcal{M}$ and $\mathcal{N}$ be the ideals of meager and null subsets of $2^{\omega}$.

Definition 1. A bijection $F: 2^{\omega} \longrightarrow 2^{\omega}$ is called an Erdös-Sierpiński mapping if

$$
X \in \mathcal{N} \Longleftrightarrow F[X] \in \mathcal{M} \text { and } X \in \mathcal{M} \Longleftrightarrow F[X] \in \mathcal{N} .
$$

Theorem 2 ([4], 1]). Assume $\mathrm{CH}$. There exists an Erdös-Sierpiński mapping.

Since the existence of Erdös-Sierpiński mapping implies that the ideals $\mathcal{M}$ and $\mathcal{N}$ have the same cardinal characteristics, the existence of such mapping cannot be proved in ZFC.

Consider the space $2^{\omega}$ as a topological group with addition modulo 2 . The following question has been attributed to Erdös and Urbanik and to Ryll-Nardzewski:

Is it consistent that there is an Erdös-Sierpiński mapping $F$ such that

$$
\forall x, y \in 2^{\omega} F(x+y)=F(x)+F(y) ?
$$

The motivation for this question is the following (see 1 for more details):

Definition 3. Suppose that $X \subseteq 2^{\omega}$. We say that $X \in \mathcal{S N}$ ( $X$ has strong measure zero) if for every set $F \in \mathcal{M}, X+F \neq 2^{\omega}$.

$X \in \mathcal{S M}$ ( $X$ is strongly meager) if for every $H \in \mathcal{N}, X+H \neq 2^{\omega}$.

An Erdös-Sierpiński mapping satisfying $F(x+y)=F(x)+F(y)$ would also map strong measure zero sets onto strongly meager sets and vice versa.

Consider the following statement (considered by Carlson in [3]):

$(\varphi)$ For every set $F \in \mathcal{M}$ there exists a set $F^{\prime} \in \mathcal{M}$ such that

$$
\forall x_{1}, x_{2} \in 2^{\omega} \exists x \in 2^{\omega}\left(\left(2^{\omega} \backslash F^{\prime}\right)+x_{1}\right) \cup\left(\left(2^{\omega} \backslash F^{\prime}\right)+x_{2}\right) \subseteq\left(2^{\omega} \backslash F\right)+x .
$$

Let $\varphi^{\star}$ be the dual statement obtained by replacing $\mathcal{M}$ with $\mathcal{N}$.

Note that $\varphi$ implies that $\mathcal{S N}$ is an ideal and $\varphi^{\star}$ implies that $\mathcal{S M}$ is an ideal (see remarks at the end of the paper).

Theorem 4 (Carlson, [3]). ZFC $\vdash \varphi$.

Received by the editors December 17, 1997 and, in revised form, April 23, 1998.

2000 Mathematics Subject Classification. Primary 03E15.

The author was partially supported by NSF grant DMS 95-05375 and the Alexander von Humboldt Foundation. 
Proof. For completeness we present a short proof based on the following classical characterization of meager sets in $2^{\omega}$ :

Theorem 5 ([1]). A set $F \subseteq 2^{\omega}$ is meager if and only if there exists a partition of $\omega$ into intervals $\left\{I_{n}: n \in \omega\right\}$ and a function $x_{F} \in 2^{\omega}$ such that

$$
F \subseteq\left\{x \in 2^{\omega}: \forall^{\infty} n x\left\lceil I_{n} \neq x_{F}\left\lceil I_{n}\right\} .\right.\right.
$$

Suppose that $F \subseteq 2^{\omega}$ is a meager set. Without loss of generality we can assume that $F=\left\{x \in 2^{\omega}: \forall^{\infty} n x\left\lceil I_{n} \neq x_{F}\left\lceil I_{n}\right\}\right.\right.$ for some partition $\left\{I_{n}: n \in \omega\right\}$ and real $x_{F} \in 2^{\omega}$.

Let $J_{n}=I_{2 n} \cup I_{2 n+1}$ for every $n$. Define

$$
F^{\prime}=\left\{x \in 2^{\omega}: \forall^{\infty} n x\left\lceil J_{n} \neq 0\right\} .\right.
$$

Suppose that $x_{1}, x_{2} \in 2^{\omega}$. Define $x=x_{1} \uparrow \bigcup_{n} I_{2 n} \cup x_{2} \uparrow \bigcup_{n} I_{2 n+1}$. It is clear that

$$
\left(\left(2^{\omega} \backslash F^{\prime}\right)+x_{1}\right) \cup\left(\left(2^{\omega} \backslash F^{\prime}\right)+x_{2}\right) \subseteq\left(2^{\omega} \backslash F\right)+x .
$$

A small modification of the above argument shows that we can consider more than two translations, countably many or even $<\operatorname{add}(\mathcal{N})$.

Theorem 6. ZFC $\vdash \neg \varphi^{\star}$.

Proof. We start with the following easy observation:

Lemma 7. Suppose that $I \subseteq \omega$ is a finite set and $J^{\prime} \subseteq J \subseteq 2^{I}$ are such that

1. $\left|J^{\prime}\right| \cdot 2^{-|I|}=1-\delta$ and $|J| \cdot 2^{-|I|}=1-\varepsilon$,

2. $\delta^{2}<\varepsilon<\delta$.

There exist $t_{1}, t_{2} \in 2^{I}$ such that

$$
\forall s \in 2^{I}\left(J^{\prime}+t_{1}\right) \cup\left(J^{\prime}+t_{2}\right) \nsubseteq J+s .
$$

Proof. Let

$$
Z=\left\{\left(t_{1}, t_{2}, z\right): z \in\left(J^{\prime}+t_{1}\right) \cup\left(J^{\prime}+t_{2}\right)\right\} .
$$

Check that for every $z \in 2^{I}$,

$$
\frac{\left|2^{I} \times 2^{I} \backslash(Z)_{z}\right|}{2^{2 \cdot|I|}}=\delta^{2}
$$

Thus $(Z)_{z} \cdot 2^{-2 \cdot|I|}=1-\delta^{2}>1-\varepsilon$ for all $z$. By the finitary version of Fubini's theorem there are $t_{1}, t_{2}$ such that

$$
\frac{\left|(Z)_{t_{1}, t_{2}}\right|}{2^{|I|}}>1-\varepsilon
$$

In particular,

$$
(Z)_{t_{1}, t_{2}}=\left(J^{\prime}+t_{1}\right) \cup\left(J^{\prime}+t_{2}\right) \nsubseteq J+s
$$

Fix a partition of $\omega$ into finite sets $\left\{I_{n}: n \in \omega\right\}$ such that $\left|I_{n}\right|>2^{n}$. For each $n$ choose $J_{n} \subseteq 2^{I_{n}}$ such that

$$
1-\frac{1}{n^{2}}+\frac{1}{n^{5}} \geq \frac{\left|J_{n}\right|}{2^{\left|I_{n}\right|}} \geq 1-\frac{1}{n^{2}} .
$$

Let

$$
F=\left\{x \in 2^{\omega}: \exists^{\infty} n x\left\lceil I_{n} \notin J_{n}\right\} .\right.
$$


The following lemma finishes the proof of Theorem 6 .

Lemma 8. For every null set $F^{\prime} \supseteq F$ there are $x_{1}, x_{2} \in 2^{\omega}$ such that for every $x \in 2^{\omega}$

$$
\left(\left(2^{\omega} \backslash F^{\prime}\right)+x_{1}\right) \cup\left(\left(2^{\omega} \backslash F^{\prime}\right)+x_{2}\right) \nsubseteq\left(2^{\omega} \backslash F\right)+x .
$$

Proof. For a closed set $C \subseteq 2^{\omega}, n \in \omega$ and $s \in 2^{<\omega}$ let

$$
C_{s}=\{x \uparrow(|s|, \omega): s \subseteq x\} \text { and } C\lceil n=\{x\lceil n: x \in C\} .
$$

Let

$$
C=\left\{x \in 2^{\omega}: \forall n x\left\lceil I_{n} \in J_{n}\right\} .\right.
$$

Without loss of generality we can assume that $C$ has positive measure. Suppose that $F^{\prime}$ is a null set. Let $C^{\prime}$ be a set of positive measure such that

$$
F^{\prime} \subseteq 2^{\omega} \backslash\left(C^{\prime}+\mathbb{Q}\right)
$$

where $\mathbb{Q}=\left\{x \in 2^{\omega}: \forall^{\infty} n x(n)=0\right\}$.

We will construct two reals $x_{1}, x_{2}$ such that for every $x$

$$
\left(C^{\prime}+x_{1}\right) \cup\left(C^{\prime}+x_{2}\right) \nsubseteq\left(2^{\omega} \backslash F\right)+x .
$$

Define by induction an increasing sequence $\left\{n_{k}: k \in \omega\right\}$ and $x_{i}\left\lceil I_{n_{k}}\right.$ for $i=1,2$. For $m \neq n_{k}$ we put $x_{i}\left\lceil I_{m}=0\right.$.

Suppose that $x_{1}\left\lceil I_{1} \cup I_{2} \cup \cdots \cup I_{n_{k}}\right.$ and $x_{2}\left\lceil I_{1} \cup I_{2} \cup \cdots \cup I_{n_{k}}\right.$ are defined. We need to define $n_{k+1}$ and $x_{i}\left\lceil I_{n_{k+1}}\right.$ for $i=1,2$. Use the Lebesgue density theorem to find sequences $\left\{r^{s}: s \in C^{\prime}\left\lceil I_{1} \cup I_{2} \cup \cdots \cup I_{n_{k}}\right\}\right.$ and $\ell>n_{k}$ such that

1. $\operatorname{dom}\left(s \frown r^{s}\right)=I_{1} \cup I_{2} \cup \cdots \cup I_{\ell}$,

2. the set $\bigcap_{s} C_{s}^{\prime} \frown r^{s}$ has positive measure.

For $m \geq \ell$ let

$$
J_{m}^{\prime}=\left\{x\left\lceil I_{m}: x \in \bigcap_{s} C_{s \frown r^{s}}^{\prime}\right\}\right.
$$

Note that

$$
\bigcap_{s} C_{s}^{\prime} \frown r^{s} \subseteq\left\{x \in 2^{\omega}: \forall m x\left\lceil I_{m} \in J_{m}^{\prime}\right\} .\right.
$$

Since the set on the left-hand side has positive measure there must be infinitely many $m$ such that

$$
\frac{\left|J_{m}^{\prime}\right|}{2^{\left|I_{m}\right|}}>1-\frac{1}{m}
$$

since $\prod_{m}\left(1-\frac{1}{m}\right)=0$. Let $n_{k+1}$ be the first such $m$ that is bigger than $\ell$. Apply the lemma to get sequences $t_{1}^{k+1}, t_{2}^{k+1}$ such that

$$
\forall s \in 2^{I_{n_{k+1}}}\left(J_{n_{k+1}}^{\prime}+t_{1}^{n_{k+1}}\right) \cup\left(J_{n_{k+1}}^{\prime}+t_{2}^{n_{k+1}}\right) \nsubseteq J_{n_{k+1}}+s .
$$

Define $x_{1}\left\lceil I_{n_{k+1}}=t_{1}^{k+1}\right.$ and $x_{2}\left\lceil I_{n_{k+1}}=t_{2}^{k+1}\right.$. This completes the definition of $x_{1}$ and $x_{2}$.

Suppose that $x \in 2^{\omega}$ is given. Let $s_{n}=x\left\lceil I_{n}\right.$. Without loss of generality we can assume

$$
\exists^{\infty} k\left(J_{n_{k+1}}^{\prime}+t_{1}^{n_{k+1}}\right) \nsubseteq J_{n_{k+1}}+s_{n_{k+1}} .
$$


Let $U \subseteq \omega$ be the set of $k$ satisfying the requirement above. We will show that

$$
C^{\prime}+x_{1} \not\left(2^{\omega} \backslash F\right)+x .
$$

For each $k$ let $u_{k} \in J_{n_{k}}^{\prime}+t_{1}^{n_{k}}$ be such that $u_{k} \in\left(J_{n_{k}}^{\prime}+t_{1}^{n_{k}}\right) \backslash\left(J_{n_{k}}+s_{n_{k}}\right)$ if possible, i.e. if $k \in U$.

Let $v_{0}=r^{\emptyset}$ and

$$
v_{k+1}= \begin{cases}v_{k} \frown u_{k / 2} & \text { if } k \text { is even, } \\ v_{k} \frown r^{v_{k}} & \text { if } k \text { is odd. }\end{cases}
$$

Let $z=\bigcup_{k} v_{k}$. Since $\left[v_{k}\right] \cap\left(C^{\prime}+x_{1}\right) \neq \emptyset$ for all $k$, it follows that $z \in C^{\prime}+x_{1}$. On the other hand $z \notin\left(2^{\omega} \backslash F\right)+x$ since

$$
\exists^{\infty} k z\left\lceil I_{n_{k}} \notin J_{n_{k}}+s_{n_{k}} .\right.
$$

Remarks. 1. The proof shows that there is no Erdös-Sierpiński mapping $F$ such that

$$
\forall X \subseteq 2^{\omega} \forall y \in 2^{\omega} \exists z \in 2^{\omega} F[X+y]=F[X]+z .
$$

2. It is consistent that $\mathcal{S M}$ is an ideal (of countable sets) ([3]). The Continuum Hypothesis implies that $\mathcal{S M}$ is not an ideal ([2]).

\section{REFERENCES}

[1] Tomek Bartoszyński and Haim Judah. Set Theory: on the structure of the real line. A.K. Peters, 1995. MR 96k:03002

[2] Tomek Bartoszyński and Saharon Shelah. Strongly meager sets do not form an ideal. to appear in Journal of Mathematical Logic.

[3] Timothy J. Carlson. Strong measure zero and strongly meager sets. Proceedings of the American Mathematical Society, 118(2):577-586, 1993. MR 94b:03086

[4] John C. Oxtoby. Measure and category, volume 2 of Graduate Texts in Mathematics. Springer Verlag, New York-Berlin, 1980. MR 81j:28003

Department of Mathematics and Computer Science, Boise State University, Boise, IDAHO 83725

E-mail address: tomek@math.idbsu.edu

$U R L:$ http://math.idbsu.edu/ ${ }^{\text {tomek }}$ 